\title{
Evaluation morphologique des spermatozoïdes
}

\author{
Rim SAIIDI1, Elodie GRUEL1, Julie ROSET-BLESSMAN1, \\ Nathalie MOUSSET-SIMEON1, Sandrine MENON1, Bertrand MACE1, Nathalie RIVES2,3
}

1 Laboratoire de Biologie de la Reproduction ; 2 Laboratoire de Biologie de la Reproduction - CECOS, Centre d'Investigation Clinique Inserm 0204 ; 3 Laboratoire de Biologie de la Reproduction - CECOS, Rouen

\section{RÉSUMÉ}

L'examen du sperme tel qu'il a été défini par l'Organisation Mondiale de la Santé (OMS) constitue une des étapes clefs dans l'exploration du couple infertile. La morphologie du spermatozoïde a été reconnue comme le meilleur facteur prédictif de la fertilité naturelle, de l'issue en insémination intra-utérine ou en fécondation in vitro classique. Les anomalies ultrastructurales des spermatozoïdes qui sembleraient être les seules anomalies capables d'influencer le devenir de l'ICSI (Intracytoplasmic Sperm Injection) ne sont pas détectables aux grossissements $\times 100$ ou x200-400 utilisés au cours de la procédure d'ICSI.

Bartoov et al. (2002) ont mis au point un système capable d'étudier la morphologie fine des spermatozoïdes mobiles en temps réel au grossissement $\times 6600$ appelé MSOME pour Magnification Motile Sperm Morphology Examination. Les anomalies détectées à ce grossissement, en dehors des anomalies morphologiques habituelles, sont des vacuoles situées sur les têtes spermatiques dont la localisation, le nombre et la taille sont variables. L'analyse en MSOME pourrait être utilisée comme facteur prédictif du pouvoir fécondant des spermatozoïdes et être réalisée dans l'exploration des atteintes spermatiques sévères avant ICSI. L'ICSI serait classique ou précédée d'une sélection fine des spermatozoïdes avant injection si les spermatozoïdes présentent un nombre élevé de vacuoles.

Mots clés : infertilité, morphologie, spermatozoïde, tératozoospermie

\section{INTRODUCTION}

L'examen du sperme (spermogramme - spermocytogramme) constitue une des étapes clefs dans l'exploration du couple infertile. L'éjaculat normal contient des spermatozoïdes présentant des variations importantes de la taille et de la forme de la tête, de l'acrosome, de la pièce intermédiaire et du flagelle. Cette hétérogénéité s'explique par le fait que le spermatozoïde, cellule hautement différenciée, est le résultat ultime d'un processus complexe : la spermatogenèse. Ce processus peut être la cible de facteurs toxiques endogènes ou exogènes qui peuvent induire la production excessive de spermatozoïdes morphologiquement anormaux responsable d'une tératozoospermie. Une morphologie anormale des spermatozoïdes peut compromettre le pouvoir fécondant d'un sperme. Cependant, le profil morphologique du sperme semble être le paramètre spermatique le plus constant chez un homme $[12,19,30,36]$.

La morphologie du sperme a été reconnue comme le meilleur facteur prédictif de la fertilité naturelle ou de l'issue en inséminations intra utérines (IIU) ou en fécondation in vitro (FIV) classique [8, 38]. Cependant, la majorité des auteurs n'ont pas retrouvé de relation entre la morphologie du sperme et le succès de l'ICSI $[20,36,31]$. Quatre explications ont été proposées. Premièrement, une morphologie normale du spermatozoïde est requise pour le passage des barrières ovocytaires qui sont court-circuitées par I'ICSI. Deuxièmement, les spermatozoïdes portant des anomalies morphologiques évidentes ne sont pas retenus pour l'injection. Troisièmement, le spermocytogramme réalisé sur des cellules randomisées de l'éjaculat n'est pas le reflet exact de la qualité du spermatozoïde injecté. Quatrièmement, les

\footnotetext{
Correspondance :

Pr Nathalie RIVES - Laboratoire de Biologie de la Reproduction CECOS, 1 rue de Germont, CHU Hôpitaux de Rouen, 76031, Rouen - Tel 0232888225 - Fax 0235982007 - Email nathalie.rives@chu-rouen.fr
} 
anomalies ultrastructurales qui sont les seules associées au devenir de I'ICSI ne peuvent pas être détectées ni au grossissement $\times 100$, ni au grossissement $\times 200-400[4,6]$.

L'analyse morphologique du sperme ou spermocytogramme est une étude descriptive de la forme des spermatozoïdes à partir d'un échantillon représentatif d'un éjaculat. La morphologie n'est qu'un des paramètres qualitatifs de la spermatogenèse. Cette analyse est dépendante des techniques d'imagerie utilisées et des critères d'interprétation adoptés pour définir un spermatozoïde morphologiquement normal.

\section{MICROSCOPIE OPTIQUE}

C'est la méthode la plus utilisée et elle fait partie des examens de routine du fait de son faible coût et de sa simplicité. L'analyse morphologique des spermatozoïdes est faite à partir de préparations fixées et colorées examinées en lumière transmise au grossissement final $\times 1000$ (objectif 100 à immersion).

\section{Méthodes de coloration}

Plusieurs colorations sont possibles : Giemsa, coloration de Papanicolaou modifiée pour les spermatozoïdes (PAP), coloration de Bruyan-Leishman ou encore coloration de Schorr. D'après certains auteurs, la coloration de PAP permet la meilleure définition de la morphologie [33]. L'Organisation Mondiale de la Santé (OMS) a souvent recommandé cette coloration [43]. La coloration de Schorr est simple à réaliser et donne de bons résultats pour une évaluation en routine [21]. Elle semble même être supérieure à la méthode de PAP modifiée pour la reconnaissance des pièces intermédiaires et des flagelles [1].

Plusieurs kits de coloration rapide ont été mis sur le marché. Cependant, ces méthodes peuvent permettre l'identification des formes normales mais ne peuvent pas nous donner les détails fournis par la coloration de PAP ou de Schorr, comme la différenciation de la pièce intermédiaire et les restes cytoplasmiques [1, 33].

Du fait de l'importance de l'impact clinique de l'analyse morphologique des spermatozoïdes, la meilleure technique doit être adoptée [1]. Une enquête du groupe de travail national sur l'assurance de qualité en Biologie de la Reproduction auprès de 214 laboratoires français avait montré que plus de cinq techniques différentes seraient utilisées pour la coloration du sperme [1]. Le choix d'une technique unique apparaît souhaitable afin d'améliorer la standardisation d'un laboratoire à un autre [1].

\section{Classifications}

Après la coloration, les spermatozoïdes sont classés en spermatozoïdes morphologiquement normaux ou anormaux. La définition d'un spermatozoïde normal est à l'origine de toutes les difficultés de l'analyse morphologique. Nombreux sont les auteurs qui se sont intéressés à ce sujet. On peut considérer aujourd'hui que les classifications des anomalies morphologiques sont aussi nombreuses que les auteurs. Cependant, trois principales classifications sont actuellement utilisées.

\section{a) Classification de David modifiée}

La classification de David [19] a été proposée en 1972 lors d'un colloque sur la fécondité et la stérilité du mâle. L'étude des spermatozoïdes récupérés dans les voies génitales féminines (en particulier dans le mucus cervical en postcoïtal) a aidé à définir les caractéristiques du spermatozoïde normal. Dans la plupart des cas, une apparente population homogène de spermatozoïdes était trouvée, contrairement à la population hétérogène trouvée dans l'éjaculat [19]. L'introduction de ces critères ajoutait un nouveau concept dans l'évaluation de la morphologie. Dans un premier temps, la description du spermatozoïde considéré comme normal était basée sur des critères biologiques sélectionnés par des principes physiologiques nécessaires à la migration à travers le mucus cervical.

Cette classification comportait initialement 13 types d'anomalies : 7 pour la tête et 6 pour le flagelle $[1,19]$. L'originalité de cette méthode reposait sur la prise en compte de toutes les anomalies observées grâce à un système de classification multiple. Un spermatozoïde porteur de plusieurs anomalies était défini par l'ensemble de ses anomalies. Ce spermatozoïde figurait plusieurs fois dans la grille de classement, pour chacune des anomalies identifiées. Depuis les années 70 , de nombreux laboratoires français ont adopté cette classification.

Au début des années 90 , cette classification a subi des modifications consistant en une redéfinition des catégories d'anomalies et l'adjonction de deux nouvelles catégories [1]. Ainsi, la catégorie tête irrégulière a été scindée en deux catégories distinguant d'une part les atypies de la région acrosomique et d'autre part les atypies de la région post acrosomique (anomalies de forme et/ou de contour et/ou de texture). La classification de David modifiée recense, en dehors des spermatozoïdes morphologiquement normaux :

- Sept anomalies de la tête : allongée, amincie, microcéphale, macrocéphale, multiple, acrosome anormal ou absent, présentant une base (région post acrosomique) anormale;

- Trois anomalies de la pièce intermédiaire : reste cytoplasmique, grêle, angulée ;

- Cinq anomalies de la pièce principale : absente, écourtée, de calibre irrégulier, enroulée et multiple.

Compte tenu de l'hétérogénéité du sperme humain et de la faible fréquence de certaines anomalies, 100 spermatozoïdes au minimum doivent être analysés pour fournir une évaluation correcte du pourcentage des spermatozoïdes dits « typiques" et des profils des atypies. Cependant, lorsque la concentration des spermatozoïdes est très faible, la classification peut être faite sur 50 spermatozoïdes, mais le résultat ne doit pas être rendu en pourcentage et la conclusion doit tenir compte de la fiabilité et de la diminution de la fréquence des anomalies retrouvées notamment lorsqu'il s'agit d'anomalies graves [1]. La fréquence normale des spermatozoïdes normaux doit être supérieure à $50 \%$ [1].

La plupart des spermatozoïdes anormaux sont porteurs d'anomalies multiples. Le nombre moyen d'anomalies par 
spermatozoïde anormal appelé index d'anomalies multiples (IAM) est un indicateur prédictif significatif du pouvoir fécondant aussi bien in vivo qu'in vitro [21]. La classification de David modifiée est largement utilisée en France y compris dans notre laboratoire.

\section{b) Classification de Kruger}

Kruger et al. [23] ont utilisé des « critères stricts " pour définir un spermatozoïde normal en évaluant la morphologie des spermatozoïdes trouvés au niveau de l'orifice interne du col après test post coïtal, comme dans la classification de David (Tableau 1). En 1990, Menkelved et al. [32, 33] ont adopté la même classification pour analyser la morphologie des spermatozoïdes fixés sur la zone pellucide dans le contexte des tests de fixation in vitro. La morphologie de ces spermatozoïdes était comparable à celle des spermatozoïdes présents au niveau de l'orifice interne du col [32].

Kruger et al. [25, 39] ont mis l'accent sur l'application clinique de l'évaluation de la morphologie. Trois groupes de sperme ont ainsi été individualisés en fonction du pourcentages de spermatozoïdes typiques :

- Formes typiques $>14 \%$ : sperme normal ;

$-4 \%<$ formes typiques < $14 \%$ : groupe G-pattern (bon pronostic) ;

- Formes Typiques < $4 \%$ : groupe $P$-pattern (mauvais pronostic).

Ces deux derniers groupes correspondent à une classification liée au pronostic en assistance médicale à la procréation (insémination ou fécondation in vitro classique). Ainsi, quand le pourcentage de formes typiques dépasse $14 \%$, les taux de fécondation sont aux alentours de $82,5 \%$ et chutent à $25 \%$ quand le taux est au dessous de $14 \%[25,39]$. Les groupes $P$ et $G$ donnent des taux de fécondation équivalents, mais le groupe $\mathrm{P}$ est associé à des taux très faibles de grossesses $[23,35]$. De plus, l'acrosome a une importance bien particulière dans la définition du spermatozoïde normal. Un index acrosomique (\% d'acrosomes normaux), avec un seuil de $8 \%$ d'acrosomes normaux, a été proposé. Quand cet index est inférieur à $15 \%$, la fertilité semble compromise $[25,33]$.

La description des spermatozoïdes typiques et atypiques précédemment faite par David est également utilisable pour décrire les anomalies dans la classification de Kruger. Cependant, la classification de Kruger recense chacune des anomalies observées individuellement alors que celle de David prend en compte toutes les anomalies observées pour un même spermatozoïde. Par ordre d'importance, l'anomalie est recensée d'abord sur l'acrosome, puis sur la tête et enfin sur la pièce intermédiaire et le flagelle. Les critères d'interprétation sont, par ailleurs, plus sévères. Tous les spermatozoïdes considérés comme «border line» (à morphologie limite) dans la classification de David sont classés par Kruger en spermatozoïdes atypiques.

\section{c) Classification de l'OMS}

L'OMS a initialement proposé sa propre classification qu'elle a améliorée au fil des éditions (Tableau 1). Cette classification a repris en partie la classification de Mac Leod publiée en 1964 [28]. Dans l'édition de 1987, seul était défini le spermatozoïde normal. Ce système ne permettait pas la prise en compte des spermatozoïdes porteurs de plusieurs anomalies [41]. En conséquence, l'un des objectifs majeur de l'édition de 1992 a été de lever l'ambiguïté sur la description du spermatozoïde normal et d'essayer d'éliminer une possible source de variation dans les interprétations (Tableau 1) [42]. La description des spermatozoïdes normaux a été détaillée. De plus, les anomalies ont été réparties en 4 catégories en fonction de leur localisation : tête, pièce intermédiaire, flagelle et reste cytoplasmique. En utilisant cette classification, toutes les formes intermédiaires étaient considérées comme anormales.

Malheureusement, les illustrations proposées ne pouvaient pas être utilisées car elles étaient en coloration monochrome et au faible grossissement. De plus, en réponse à l'étude de Ducot et al. [21], I'IAM devient le TZP (index de tératozoospermie) qui est la moyenne du nombre d'anomalies par spermatozoïde anormal [33]. Barratt et al. [3] ont comparé la signification clinique de la morphologie des spermatozoïdes évalués par les critères de l'OMS de 1987 et de 1992. Ils ont conclu que l'édition de 1992 n'apportait pas de changement majeur dans la valeur prédictive de ce paramètre pour déterminer la fertilité du patient. Les critères définis par l'OMS n'ont pas été associés à des tests fonctionnels des spermatozoïdes et, de ce fait, n'ont pas permis d'améliorer la signification clinique de l'évaluation de la morphologie des spermatozoïdes.

Dans son édition de 1999 [43], l'OMS a décidé d'adopter les stricts critères de Kruger. Le TZP est considéré comme un paramètre optionnel. Le reste cytoplasmique n'était pas inclus dans le calcul du TZP. Cette édition n'était qu'une intégration de l'édition de 1992 et de la classification de Kruger. Les changements les plus importants étaient le seuil bas (14\%) des formes normales et la redéfinition de ces chiffres comme valeur de référence et non pas comme valeur normale [43].

\section{d) Difficultés rencontrées lors de la réalisation d'un spermocytogramme : le spermocytogramme automatisé}

Après avoir défini les méthodes de coloration et les différentes classifications adoptées dans la réalisation du spermocytogramme, il apparaît clair que cette analyse présente sous une apparente simplicité, de réelles difficultés d'interprétation [1] tenant essentiellement de :

- l'utilisation de systèmes de classification multiples,

- la difficulté à définir le spermatozoïde normal,

- du caractère subjectif de cette analyse morphologique responsable d'une variabilité très importante des résultats entre les laboratoires, et, au sein d'un même laboratoire, entre les observateurs.

Pour toutes ces raisons, l'analyse automatisée. de la morphologie semble être une approche intéressante supprimant le caractère subjectif de la mesure et réduisant ainsi les erreurs d'interprétation. Ainsi, plusieurs systèmes d'analyse d'image ont été développés se basant sur les paramètres morphologiques préalablement définis. 
Tableau 1 : Comparaison des principales classifications morphologiques des spermatozoïdes selon l'OMS.

\begin{tabular}{|c|c|c|c|c|}
\hline & $\begin{array}{l}\text { OMS } 1987 \\
{[40]}\end{array}$ & $\begin{array}{l}\text { OMS } 1992 \\
{[41]}\end{array}$ & $\begin{array}{l}\text { David } \\
\text { [18] }\end{array}$ & $\begin{array}{l}\text { Kruger } \\
\text { [24] }\end{array}$ \\
\hline $\begin{array}{l}\text { Nombre de } \\
\text { spz examinés }\end{array}$ & 100 & Préférence 200 & 100 & 200 \\
\hline Tête & & & & \\
\hline Forme & $\begin{array}{l}\text { Contour ovalaire } \\
\text { Contour régulier }\end{array}$ & $\begin{array}{l}\text { Contour ovalaire } \\
\text { Têtes picnotiques non } \\
\quad \text { considérées } \\
\text { Spz intermédiaires anormaux }\end{array}$ & $\begin{array}{l}\text { Contour ovalaire } \\
\text { Contour régulier } \\
\text { Texture homogène } \\
7 \text { anomalies }\end{array}$ & $\begin{array}{l}\text { Contour ovalaire } \\
\text { Contour régulier } \\
\text { Spz intermédiaires } \\
\text { anormaux }\end{array}$ \\
\hline $\begin{array}{l}\text { Taille } \\
\text { longueur } \\
\text { Largeur }\end{array}$ & $\begin{array}{l}3-5 \mu \mathrm{m} \\
2-3 \mu \mathrm{m}\end{array}$ & $\begin{array}{l}4-5,5 \mu \mathrm{m} \\
2.5-3,5 \mu \mathrm{m}\end{array}$ & $\begin{array}{l}3-5 \mu \mathrm{m} \\
3 \mu \mathrm{m}\end{array}$ & $\begin{array}{l}5-6 \mu \mathrm{m} \\
2,5-3,5 \mu \mathrm{m}\end{array}$ \\
\hline Acrosome & $\begin{array}{l}>1 / 3 \text { surface de } \\
\text { la tête }\end{array}$ & $40-70 \%$ surface de la tête & $\begin{array}{l}40-70 \% \text { de la surface } \\
\text { de la tête }\end{array}$ & $\begin{array}{l}40-70 \% \text { de la surface } \\
\text { de la tête }\end{array}$ \\
\hline Vacuoles & Aucun détail & $<20 \%$ surface tête & Aucun détail & Aucun détail \\
\hline $\begin{array}{l}\text { Pièce } \\
\text { intermédiaire }\end{array}$ & $\begin{array}{l}\text { Fine } \\
\text { Régulière } \\
\text { Rectiligne } \\
\text { Alignée avec } \\
\text { l'axe longitudinal } \\
\text { de la tête } \\
\text { I: } 7-8 \mu \mathrm{m} \\
<1 / 3 \mathrm{~L} \text { de la tête } \\
\end{array}$ & $\begin{array}{l}\text { Aucune définition de la pièce } \\
\text { intermédiaire dite normale }\end{array}$ & $\begin{array}{l}\text { Alignée avec l'axe } \\
\text { longitudinal de la tête } \\
1 \text { à } 2 \times I \text { de la tête } \\
\text { Diamètre } 0,6-0,8 \mu \mathrm{m} \\
3 \text { anomalies }\end{array}$ & $\begin{array}{l}\text { Fine } \\
\\
I: 1,5 \times \mid \text { de la tête } \\
L<1 \mu \mathrm{m} \\
\end{array}$ \\
\hline $\begin{array}{l}\text { Reste } \\
\text { cytoplasmique }\end{array}$ & Aucun détail & $<1 / 3$ tête & $<1 / 3$ tête & $<30 \%$ tête \\
\hline Flagelle & $\begin{array}{l}\text { Fin } \\
\text { Régulier } \\
1>45 \mu \mathrm{m}\end{array}$ & $\begin{array}{l}\text { Aucune dimension } \\
\text { Aucune description du flagelle } \\
\text { normal }\end{array}$ & $\begin{array}{l}\text { Régulier } \\
\mathrm{I}: 45 \mu \mathrm{m}(10 \times \mathrm{I} \text { tête }) \\
\mathrm{L}: 0,3-0,5 \mu \mathrm{m}\end{array}$ & $\begin{array}{l}\text { Régulier } \\
1: 45 \mu \mathrm{m}\end{array}$ \\
\hline $\begin{array}{l}\text { Seuil de } \\
\text { normalité } \\
\text { (\% de spz } \\
\text { typiques) }\end{array}$ & $50 \%$ & $30 \%$ & $50 \%$ & $14 \%$ \\
\hline Index d'anomalies & & TZP & IAM & \\
\hline
\end{tabular}

IAM : index d'anomalies multiples ; $L$ = largeur, $I=$ Iongueur ; OMS : Organisation Mondiale de la Santé ; spz = spermatozoïdes ; TZP = Index de tératozoospermie. 
L'ASMA (Automated Sperm Morphology Analysis) peut ainsi être programmé suivant n'importe quelle classification préexistante. Mais l'inconvénient de ce système est que seule la tête spermatique est prise en considération. La pièce intermédiaire et le flagelle sont des structures de petite taille faiblement colorées rendant leurs identifications difficiles par l'analyseur. De ce fait, la fréquence des spermatozoïdes anormaux chute de $10 \%$ par rapport à l'observation manuelle. L'ASMA donne une reproductibilité satisfaisante avec un temps d'observation de $10 \mathrm{mn}$ pour examiner 100 spermatozoïdes [27].

Un autre système a été proposé, IVOS (Hamilton, Thorne Research, Beverly, MA), qui évalue seulement la forme, la taille de la tête et l'acrosome. Coetzee et al. ont trouvé que l'analyse morphologique par IVOS avait une valeur prédictive des chances de succès en fécondation in vitro [17].

Malgré l'objectivité de ces méthodes automatisées, une standardisation de la préparation des lames est indispensable. Un consensus sur les critères sélectifs retenus pour identifier un spermatozoïde normal doit être défini. De plus, le développement d'un système capable de bien étudier le flagelle semble important.

En conclusion, malgré les efforts réalisés dans la standardisation de l'évaluation de la morphologie des spermatozoïdes en microscopie optique, son appréciation varie au gré des classifications et constitue un facteur limitant dans la détermination objective des seuils de normalité ou d'anormalité. Le recours à d'autres méthodologies semble donc indispensable pour améliorer le pouvoir discriminant de la morphologie dans la distinction des spermatozoïdes fécondants et non fécondants.

\section{MICROSCOPIE ÉLECTRONIQUE}

La microscopie optique permet une bonne évaluation de la mobilité, de la numération et de la morphologie des spermatozoïdes. Elle permet uniquement de faire une description des surfaces et ne donne aucune information précise sur les organites et le noyau spermatique qui jouent un rôle important dans la fertilité. Un spermatozoïde peut être apparemment normal en microscopie optique utilisant les colorations conventionnelles avec cependant un noyau dont la chromatine est mal condensée ou fragmentée [4]. De ce fait, certains auteurs se sont intéressés à l'étude ultrastructurale des spermatozoïdes afin de déterminer l'organisation et l'intégrité des différentes structures, en utilisant la microscopie électronique (ME) à transmission (TEM) et à balayage (SEM).

La TEM permet de visualiser des sections de spermatozoïde avec une image en double dimension et des détails des structures ultrafines : l'acrosome, le post acrosome, le cytoplasme et l'intégrité des différents composants internes du flagelle comme l'axonème et les structures périaxonémales à un grossissement $\times 35000$. Cependant, la structure tridimensionnelle globale du spermatozoïde n'est pas visualisée. Cette limitation est remédiée par la SEM. Le morphogramme à balayage est obtenu en attachant une particulière attention à la tête et au squelette du flagelle [4]. Bartoov et al. s'intéressent aux anomalies structurales des différents composants du spermatozoïde en combinant les résultats des analyses en TEM (acrosome, post-acrosome, flagelle) et en SEM (taille et forme de la tête) [4].

Pour rendre cette analyse ultrastructurale utilisable en clinique, afin de différencier les hommes fertiles et infertiles, Bartoov et al. ont établi un index quantitatif ultrastructural appelé QUM (Quantitative UltraMorphological index)

$$
\begin{gathered}
\text { QUM }=\begin{array}{c}
(\% \text { des noyau } \times \text { normaux }) \times 0,04-(\% \text { des } \\
\text { acrosomes malformés } \times 0,032)
\end{array} \\
-(\% \text { des fibres denses malformées } \times 0,044)-0,07
\end{gathered}
$$

Cet index peut être considéré comme facteur prédictif positif de la fertilité dans $75 \%$ des cas. En combinant le QUM avec les paramètres conventionnels du sperme, cette valeur prédictive positive atteint $80 \%$. Cette technique coûteuse et onéreuse n'est pas disponible dans tous les laboratoires d'analyse du sperme. Le QUM peut être utile pour les patients qui sont en échec d'insémination ou de fécondation in vitro malgré des paramètres spermatiques conventionnels normaux [4].

En conclusion, l'accessibilité actuelle à l'analyse en microscopie électronique est de plus en plus limitée par disparition progressive des plates formes pratiquant cette analyse et par défaut d'experts biologistes dans ce domaine. L'analyse en TEM doit donc être réservée aux tératozoospermies monomorphes ou spécifiques et ne doit pas être proposée dans les tératozoospermies polymorphes. De plus, le QUM décrit par Bartoov et al. [4] n'a, jusqu'à ce jour, pas été utilisé par d'autres équipes que la sienne et a priori ne constitue plus pour cette équipe une méthode d'évaluation de routine.

\section{OBSERVATION DES SPERMATOZOÏDES MOBILES AU GROSSISSEMENT X6600}

Toutes les techniques d'évaluation morphologique des spermatozoïdes qui ont été décrites ci-dessus nécessitent une fixation de ces derniers et ne permettent pas une étude en temps réel des spermatozoïdes vivants et mobiles.

En 2002, Bartoov et al. ont mis au point un système optique appelé MSOME, pour High Magnification Motile Sperm Morphology Examination, qui permet d'examiner le spermatozoïde en temps réel à fort grossissement [6]. Pour cela, ils utilisent un microscope inversé équipé d'un contraste interférentiel de Nomarski et d'un objectif 100 à immersion. Ce microscope est équipé d'une caméra vidéo 3D et d'un moniteur couleur [6]. Le grossissement final qui tient compte de l'objectif du microscope, du zoom vidéo et du moniteur est de $\times 6600$. Les spermatozoïdes sont disposés dans une boite de pétri à fond de verre. Les spermatozoïdes mobiles sont observés à fort grossissement en bordure de goutte et les différentes parties du spermatozoïde comme l'acrosome, le post acrosome, le cou, la pièce intermédiaire, le flagelle et le noyau sont analysées.

Un spermatozoïde normal a une tête ovale, lisse, symétrique, 
sa taille est comprise entre $4,75 \pm 0,28 \mu \mathrm{m}$ pour la longueur et $3,28 \pm 0,2 \mu \mathrm{m}$ pour la largeur. Ces critères ont été définis arbitrairement par rapport aux études anciennes d'examination des spermatozoïdes en ME. La chromatine est considérée comme anormale si une ou plusieurs vacuoles occupent plus de $4 \%$ de la surface totale de la tête (Figure 1). Une tête normale doit avoir une forme normale et un contenu normal $[4,6]$. Les anomalies morphologiques spécifiques retrouvées en MSOME sont récapitulées dans le Tableau 2 [6].

En conclusion, le MSOME constitue une alternative au SEM dans l'évaluation de la morphologie fine du spermatozoïde. Son accessibilité tend actuellement à se développer en France. Les anomalies détectées par le MSOME sont cependant variables, les critères descriptifs définis par Bartoov et al. [6] ne sont pas transposables d'emblée dans un laboratoire pour une analyse de routine. Ainsi, il semble utile de définir un spermocytogramme MSOME afin d'établir les limites entre les spermes normaux et les spermes anormaux, le but étant de trouver une éventuelle relation entre les paramètres spermatiques conventionnels et ceux analysés par le MSOME. II est possible que les laboratoires soient à nouveau confrontés à la nécessité de définir une nouvelle classification de ces anomalies proche ou éloignée de celle de Bartoov [6].

\section{IMPACT DE LA MORPHOLOGIE DES SPERMATOZOIDES SUR LA FERTILITÉ}

La question des altérations de la fertilité masculine est sans cesse reposée. L'évaluation du potentiel de fertilité masculine se base principalement sur l'examen du spermogramme. La morphologie, le paramètre le plus constant du sperme, figure parmi les moyens les plus fiables de cette évaluation. Les structures d'un spermatozoïde normal lui procure la meilleure organisation pour assurer ses fonctions spécifiques. La tératozoospermie est une combinaison d'anomalies morphologiques entraînant une altération des fonctions spermatiques.

Matalliotakis et al. [30] ont montré que la fertilité masculine est compromise de façon différente en fonction des anomalies spermatiques observées. En effet, les anomalies de la tête et de l'acrosome semblent être les plus graves. D'après certains auteurs, l'évaluation de la morphologie selon des critères stricts apparaît comme le paramètre le plus informatif pour différencier les hommes fertiles et infertiles [22]. Slama et al. [36] ont étudié la corrélation entre le délai de conception et les paramètres du sperme. Ils ont observé une diminution de ce délai quand la proportion de spermatozoïdes normaux était supérieure à $39 \%$ selon la classification de David et à $19 \%$ selon la classification de Kruger. Le délai de conception est significativement corrélé à l'IAM sur toutes les valeurs sans aucun profil clair ou valeur discriminative [36]. Les anomalies chromosomiques de nombre des spermatozoïdes, ou aneuploïdies, sont plus fréquentes en cas de tératospermie majeure et monomorphe. Ces anomalies chromosomiques peuvent retentir sur le développement embryonnaire et favoriser la survenue de fausses couches $[34,40]$.

L'exploration morphologique des spermatozoïdes est l'élément clé pour étudier le potentiel de fertilité d'un homme, et le facteur pronostic de chance de succès le plus fiable en AMP [26]. La fécondation in vitro a mis en évidence l'importance de l'acrosome et du noyau spermatique dans le pouvoir fécondant du spermatozoïde. Par exemple, un spermatozoïde sans acrosome ne fécondera qu'en ICSI. Les spermatozoïdes acéphaliques ne sont pas fécondants quelque soit la méthode de procréation assistée utilisée. En cas de dyskinésie flagellaire, le spermatozoïde ne peut pas pénétrer la zone pellucide et seul l'ICSI pourrait être envisageable. Cependant, des anomalies du centriole peuvent être associés et être responsables d'un arrêt de développement embryonnaire par altération du fuseau de division mitotique [16].

D'après Kruger, en FIV conventionnelle, si le taux de spermatozoïdes normaux est inférieur à $14 \%$, le taux de fécondation est de $37 \%$ alors que ce taux atteint $85 \%$ quand les formes normales dépassent 14\% [25]. En ICSI, cette relation entre la morphologie et le pouvoir fécondant n'est pas vérifiée car le critère ultime de la réussite d'une ICSI est la présence d'au moins un spermatozoïde vivant normal par ovocyte [31]. Cependant, la relation potentielle entre la forme des spermatozoïdes et leur intégrité génétique est devenue pertinente depuis l'introduction de l'ICSI, car dans cette procédure, la sélection ne se base pas sur l'interaction entre le spermatozoïde et la zone pellucide, comme dans la FIV, mais sur la visualisation de la forme du spermatozoïde et de ses anomalies par le biologiste. Cependant, le spermatozoïde apparemment normal peut être diploïde ou disomique [15, 35].

En dehors des anomalies chromosomiques, la qualité nucléaire et probablement cytoplasmique du spermatozoïde peut altérer les chances de succès de l'ICSI. En effet, les altérations de l'ADN spermatique peuvent retentir sur le développement des embryons et leur chance d'implantation. Un taux élevé d'ADN spermatique dénaturé ( $>27 \%$ ) évalué par SCSA, pour Sperm Chromatin Structure Assay, se traduit par une absence de survenue de grossesse [26].

Ainsi, une évaluation objective et pertinente des altérations morphologiques des spermatozoïdes s'impose mais n'est pas simple à mettre en œuvre. Deux raisons principales peuvent être retenues pour expliquer cette difficulté : les spermatozoïdes présentent une hétérogénéité structurale importante, et le biologiste ne peut pas être sûr que le spermocytogramme des spermatozoïdes éjaculés reflète véritablement la qualité des spermatozoïdes qui sont capables de féconder l'ovocyte [6].

Le premier problème peut être résolu par une classification morphologique des spermatozoïdes selon les différentes anomalies qu'ils présentent [4]. L'analyse ultrastructurale des spermatozoïdes par la ME a la valeur prédictive la plus importante pour évaluer le pouvoir fécondant des spermatozoïdes [16]. La seconde difficulté peut être résolue par l'examen morphologique fin des spermatozoïdes mobiles sélectionnés. Le MSOME combine ces deux solutions puisque l'analyse s'effectue à une échelle proche de l'ultrastructure et en temps réel des seuls spermatozoïdes mobiles [6].

Les anomalies détectées par le MSOME, en dehors des anomalies morphologiques habituelles, sont des vacuoles situées sur les têtes spermatiques dont la localisation, le 
Tableau 2 : Malformations spécifiques du spermatozoïde observées en MSOME (High Magnification Motile Sperm Morphology Examination), d'après [6].

\begin{tabular}{|c|c|c|c|c|c|c|c|}
\hline & Acrosome & $\begin{array}{c}\text { Post } \\
\text { acrosome }\end{array}$ & No) & & Cou & Flagelle & Mitochondrie \\
\hline 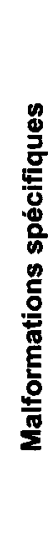 & $\begin{array}{l} \\
\text { Absent } \\
\text { Partiel }\end{array}$ & 更 & $\begin{array}{l}\text { Forme } \\
\\
\text { Microcéphale ovalaire } \\
\text { Macrocéphale ovalaire } \\
\text { Mince (L < 2,9 um) } \\
\text { Large (I } \geq 3,7 \text { um) } \\
\text { Court (L < 4,2 um) } \\
\text { Irrégulier }\end{array}$ & $\begin{array}{l}\text { Surface } \\
\text { vacuolaire } \\
>4 \% \text { de la } \\
\text { surface de la } \\
\text { tête }\end{array}$ & $\begin{array}{l}\text { Désaxé } \\
\text { Irrégulier } \\
\text { Reste } \\
\text { cytoplasmique }\end{array}$ & $\begin{array}{l}\text { Absent } \\
\text { Angulé } \\
\text { Irrégulier } \\
\text { Multiple } \\
\text { Court }\end{array}$ & $\begin{array}{l}\text { Absente } \\
\text { Partielle } \\
\text { Désorganisée }\end{array}$ \\
\hline
\end{tabular}

= largeur; $I=$ longueur
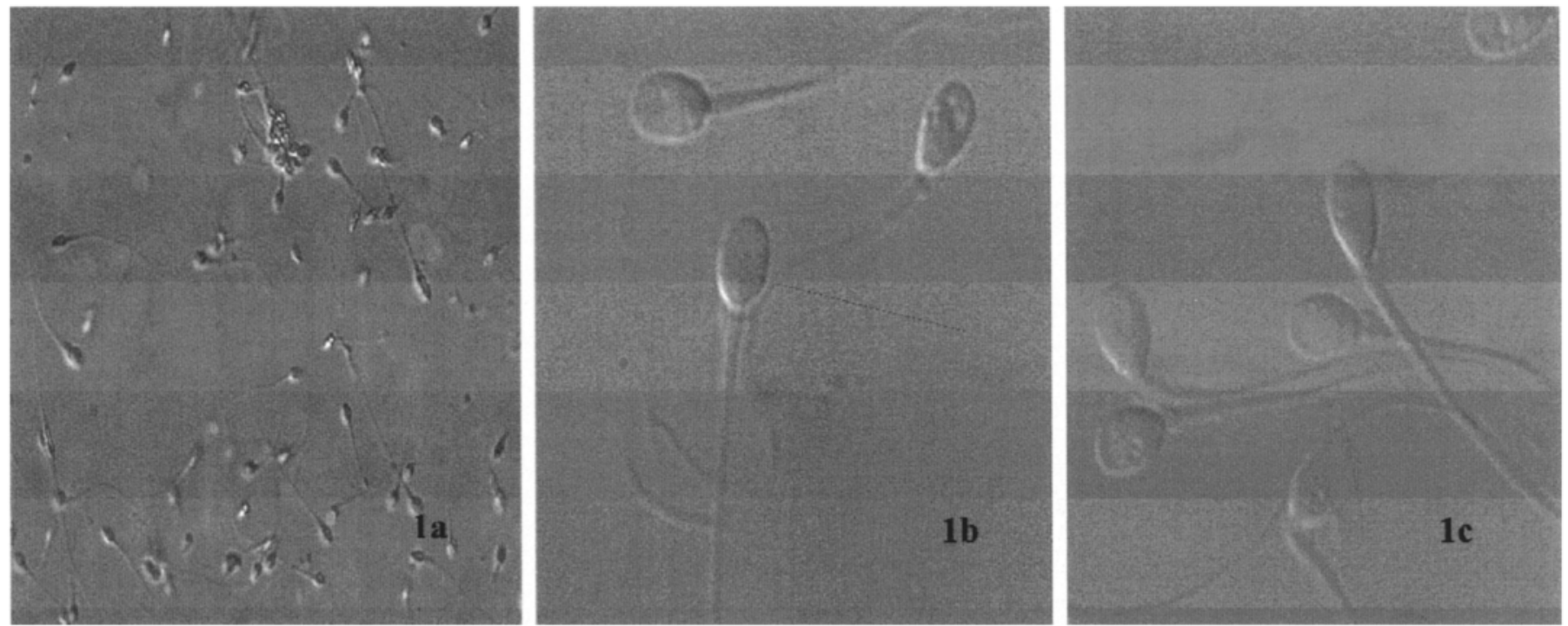

Figure 1 : Spermatozoïdes observés au microscope inversé (grossissement $x 200$ : photographie 1a) et au grossissement x6600 (MSOME = High Magnification Motile Sperm Morphology Examination): photographies $1 \mathrm{~b}$ et $1 \mathrm{c}$ avec des spermatozoïdes présentant une ou plusieurs vacuoles céphaliques. 
nombre et la taille sont variables. Boughali et al. [13] retrouvent une valeur seuil de $8 \%$ de spermatozoïdes à morphologie fine normale évaluée en MSOME au dessous de laquelle un échec complet de fécondation en FIV est observée dans $40 \%$ des cas. Les études de Bartoov et al. [6] et de Berkovitz et al. [10] ont montré que plus la surface vacuolaire était élevée, plus les taux d'implantation et de grossesse étaient faibles en ICSI, plus le taux de fausses couches augmentait probablement par altération spermatique. Bartoov et al. et Berkovitz et al. supposent que ces vacuoles sont probablement d'origine nucléaire, en se basant sur les données de la ME $[6,9]$. L'aspect du noyau spermatique observé par le MSOME reflète la qualité de l'ADN spermatique qui a un impact important sur le développement embryonnaire et le succès de l'ICSI [6, 7]. Cette idée concorde avec l'étude de Larson et al. [26] qui ont conclu que l'intégrité de la chromatine spermatique apparaît comme cause potentielle d'échec en ICSI. Ainsi, $62 \%$ des couples en échec d'ICSI conçoivent après une tentative d'IMSI sans augmentation du taux de fécondation.

Bartoov et al. [7] supposent l'existence possible d'une relation entre les anomalies spermatiques chromosomiques responsables du défaut d'implantation embryonnaire ou de fausses couches ultraprécoces et le statut nucléaire évalué par le MSOME. En effet, dans une étude portant sur 24 couples présentant une infécondité masculine, l'ICSI a été réalisée avec des spermatozoïdes sélectionnés au grossissement x6600 avec un noyau normal. Les femmes étaient âgées de moins de 37 ans et avaient eu au moins 5 échecs d'ICSI. Aucune différence significative n'a été notée concernant le taux de fécondation par cycle. Trois patients n'avaient aucun noyau spermatique normal et aucune grossesse n'est survenue au sein de ces couples. Un taux de grossesse de $67 \%$, un taux d'implantation de $47 \%$ et un taux de fausses couches de $5 \%$ ont été obtenus. En considérant que la chance d'obtenir une grossesse après 5 tentatives d'ICSI est de $3 \%$, les altérations morphologiques fines du noyau spermatique semble directement liées à la survie embryonnaire après ICSI. La technique IMSI, pour Intracytoplasmic Morphogically Selected Sperm Injection, venait d'être définie [5]. Dans une étude prospective portant sur 100 couples ayant eu une ICSI classique, 100 spermatozoïdes mobiles et d'apparence normale ont été analysés par le MSOME. Au total, 33\% des spermatozoïdes et seulement $26,8 \%$ des têtes spermatiques étaient normaux. Seule la morphologie du noyau spermatique était significativement corrélée au taux de fécondation et de grossesse en ICSI [6]. Bartoov et al. [7] sélectionnent 50 couples après deux échecs d'ICSI d'indication masculine et effectuent une IMSI en injectant uniquement des spermatozoïdes de type 0 (sans vacuole). Le taux d'implantation est ainsi multiplié par 3 et le taux de fausses couches précoces est considérablement réduit.

De Vos et al., en 2003, évaluent, dans une étude rétrospective, l'influence de la morphologie du spermatozoïdes injecté au grossissement $x 400$ sur le taux de fécondation et sur le taux de grossesse. Lorsqu'un spermatozoïde anormal est injecté, le taux de fécondation chute de $72 \%$ à $60 \%$. Aucune différence significative n'a été observée concernant la morphologie des embryons observés à $\mathrm{J} 2$ ou à $\mathrm{J} 3$, cependant, une diminution du taux de grossesse de $20 \%$ à $36,7 \%$ et du taux d'implantation de $19 \%$ à $10 \%$ a été notée. Aucune information sur la capacité de développement en blastocyste de ces embryons n'a été mentionnée. Ainsi, un spermatozoïde apparemment normal peut présenter des anomalies dissimulées capables d'empêcher la survenue d'une grossesse [20]. Cependant, une étude récente portant sur 25 tentatives d'ICSI sans sélection d'indication ne retrouve pas de valeur prédictive de la morphologie en MSOME sur le taux de fécondation, la qualité embryonnaire ou l'issue de l'ICSI. La présence de vacuoles ne semble pas avoir de signification péjorative pour l'obtention d'une grossesse. Cependant, les résultats de cette étude se fondent sur un faible effectif de tentatives d'ICSI [37]. Cassuto et al. et Bach et al. ont mis en évidence que les larges vacuoles réduisent dramatiquement la capacité de l'embryon à atteindre le stade blastocyste et que la surface vacuolaire est corrélée négativement à la qualité du blastocyste obtenu [2, 14]. En 2005, Berkovitz et al. ont défini des critères de choix des spermatozoïdes injectés en utilisant le MSOME. Les spermatozoïdes de premier choix sont les spermatozoïdes de taille normale et ayant une chromatine normale ( 0 ou 1 vacuole occupant moins de $4 \%$ de la surface de la tête). Les spermatozoïdes de deuxième choix sont par ordre de préférence : (i) spermatozoïdes avec une chromatine normale et une taille anormale mais de forme ovale, (ii) spermatozoïdes avec une chromatine normale mais de forme non ovale, (iii) spermatozoïdes avec une chromatine anormale avec de larges vacuoles. Les spermatozoïdes de premier choix permettent un taux de fécondation de $70 \%$, un taux d'implantation de $25 \%$ et un taux de fausses couches de $5 \%$. Quant aux spermatozoïdes de deuxième choix, le taux de fécondation est de $50 \%$, d'implantation de $6 \%$ et de fausses couches de $57 \%$. Les larges vacuoles du noyau spermatique semblent indiquer un dommage important de I'ADN plus que la forme ou la taille de la tête spermatique [9].

Le spermocytogramme MSOME pourrait constituer un préalable dans l'exploration des atteintes spermatiques afin d'être utilisé comme facteur prédictif des chances de succès des techniques d'AMP et de proposer le mode d'AMPle plus adapté en fonction des altérations spermatiques observées. Une IMSI pourrait être d'emblée réalisée en présence d'un taux particulièrement élevé (dont le seuil reste à définir) de spermatozoïdes présentant de larges vacuoles détectées en MSOME.

\section{RÉFÉRENCES}

1. AUGER J., EUSTACHE F. : Standardisation de la classification morphologique des spermatozoïdes humains selon la méthode de David modifiée. Andrologie, 2000, 10 : 358-373.

2. BACH M., NEYER A., STECHER A. et al. : Morphological integrity of human sperm nuclei and blastocyst formation after intracytoplasmic morphologically selected sperm injection (IMSI). The $23^{\text {rd }}$ Annual Meeting of the ESHRE, Lyon, 2007, France.

3. BARRATT C.L., NAEENI M., CLEMENTS S., COOKE I.D. : Clinical value of sperm morphology for in-vivo fertility : comparison between World Health Organization criteria of 1987 and 1992. Hum. Reprod., 1995, $10: 587-593$

4. BARTOOV B., ELTES F., PANSKY M., LANGZAM J., REICHART M., 
SOFFER Y. : Improved diagnosis of male fertility potential via a combination of quantitative ultramorphology and routine semen analyses. Hum. Reprod., 1994, 9 : 2069-2075.

5. BARTOOV B., BERKOVITZA., ELTES F. : Selection of spermatozoa with normal nuclei to improve the pregnancy rate with intracytoplasmic sperm injection. N. Engl. J. Med., 2001, 345 : 1067-1068.

6. BARTOOV B., BERKOVITZ A., ELTES F., KOGOSOWSKI A., MENEZO Y., BARAKY. : Real-time fine morphology of motile human sperm cells is associated with IVF-ICSI outcome. J. Androl., 2002, $23: 1-8$

7. BARTOOV B., BERKOVITZA., ELTES F. et al. : Pregnancy rates are higher with intracytoplasmic morphologically selected sperm injection than with conventional intracytoplasmic injection. Fertil. Steril., 2003, $80: 1413-1419$.

8. BERKOVITZA., ELTES F., SOFFER Y. et al. : ART success and in vivo sperm cell selection depend on the ultramorphological status of spermatozoa. Andrologia, 1999, $31: 1-8$

9. BERKOVITZ A., ELTES F., YAARI S. et al. : The morphological normalcy of the sperm nucleus and pregnancy rate of intracytoplasmic injection with morphologically selected sperm. Hum. Reprod., 2005, $20: 185-190$.

10. BERKOVITZ A., ELTES F., LEDERMAN H. et al : How to improve IVF-ICSI outcome by sperm selection. Reprod. Biomed. Online., 2006, $12: 634-638$.

11. BERKOVITZA., ELTES F., ELLENBOGENA., PEER S., FELDBERG D., BARTOOV B. : Does the presence of nuclear vacuoles in human sperm selected for ICSI affect pregnancy outcome ? Hum. Reprod. 2006, $21: 1787-1790$.

12. BISSON J.P., DAVID G. : Structure du spermatozoïde humain Contracept. Fertil. Sex., 1976, $4: 117-124$.

13. BOUGHALI H., WITTEMER C.H., VIVILLE S. : Intérêt de l'analyse de la morphologie fine et de la qualité nucléaire des spermatozoïdes dans le cadre des techniques d'AMP. Andrologie, 2006, $16: 38-45$

14. CASSUTO G.N., PLOUCHART J.M., BALET R., LARUE L., BOURET D. : Interest of a new morphological classification of human spermatozoa for ICSI allowing to obtain a better blastocyst score. The $23^{\text {rd }}$ Annual Meeting of the ESHRE, Lyon, 2007, France.

15. CELIK-OZENCI C., JAKAB A., KOVACS T. et al. : Sperm selection for ICSI : shape properties do not predict the absence or presence of numerical chromosomal aberrations. Hum. Reprod., 2004, 19 : 2052-2059.

16. CHEMES H.E., RAWE V.Y. : Sperm pathology : a step beyond descriptive morphology. Origin, characterization and fertility potential of abnormal sperm phenotypes in infertile men. Hum. Reprod. Update, $2003,9: 405-428$.

17. COETZEE K., DE VILLIERS A., KRUGER T.F., LOMBARD C.J. : Clinical value of using an automated sperm morphology analyzer (IVOS). Fertil. Steril., 1999, $71: 222-225$.

18. DALZELL L.H., MCVICAR C.M., MCCLURE N., LUTTON D., LEWIS S.E. : Effects of short and long incubations on DNA fragmentation of testicular sperm. Fertil. Steril., 2004, 82 : 1443-1445.

19. DAVID G., BISSON J.P., CZYGLIK F., JOUANNET P., GERNIGNON C. : Anomalies morphologiques du spermatozoïde humain, proposition pour un système de classification. J. Gyn. Obst. Biol. Reprod., 1975, $4: 17-36$.

20. DE VOS A., VAN DE VELDE H., JORIS H., VERHEYEN G., DEVROEY P., VAN STEIRTEGHEMA. : Influence of individual sperm morphology on fertilization, embryo morphology, and pregnancy outcome of intracytoplasmic sperm injection. Fertil. Steril., 2003, 79: $42-48$.

21. DUCOT B., SPIRAA., FENEUX D., JOUANNET P. : Male factors and the likelihood of pregnancy in infertile couples. II. Study of clinical characteristics-practical consequences. Int. J. Androl., 1988, 11 : 395-404.

22. GUZICK D.S., OVERSTREET J.W., FACTOR-LITVAK P. et al. :
Sperm morphology, motility, and concentration in fertile and infertile men. N. Engl. J. Med., 2001, 345 : 1388-1393.

23. HAMMADEH M.E., STREHLER E., ZEGINIADOU T., ROSENBAUM P., SCHMIDT W. : Chromatin decondensation of human sperm in vitro and its relation to fertilization rate after ICSI. Arch. Androl., 2001, 47: 83-87.

24. JUNCAA.M., COHEN-BACRIE P., HAZOUTA. : ICSI : peut-on choisir les spermatozoides ? J. Gynecol. Obstet. Biol. Reprod., 2005, 34 : $1 S 31-1 \$ 34$.

25. KRUGER T.F., MENKVELD R., STANDER F.S. et al. : Sperm morphologic features as a prognostic factor in in vitro fertilization. Fertil. Steril., 1986, $46: 1118-1123$.

26. LARSON K.L., DEJONGE C.J., BARNESA.M., JOST L.K., EVENSON D.P. : Sperm chromatin structure assay parameters as predictors of failed pregnancy following assisted reproductive techniques. Hum. Reprod., 2000, $15: 1717-1722$.

27. LE LANNOU D. : L'analyse automatisée de la morphologie du spermatozoïde. Andrologie, 1997, $7: 427-432$.

28. MAC LEOD J. : Human Seminal Cytology as a sensitive indicator of the germinal epithelium. Int. J. Fertil., 1964, $9:$ 281-295.

29. MASHIACH R., FISCH B., ELTES F., TADIR Y., OVADIA J., BARTOOV $B$. : The relationship between sperm ultrastructural features and fertilizing capacity in vitro. Fertil. Steril., 1992, $57: 1052-1057$.

30. MATALLIOTAKIS I., PANIDIS D., VLASSIS G., KALOGEROPOULOS A., ZOLINDAKI A., KOUMANTAKIS $E$. : The evaluation of morphological abnormalities of human spermatozoa in fertile and infertile men. Eur. J. Obstet. Gynecol. Reprod. Biol., 1996, 66 : 6568.

31. MCKENZIE L.J., KOVANCI E., AMATO P., CISNEROS P., LAMB D., CARSON S.A. : Pregnancy outcome of in vitro fertilization/ intracytoplasmic sperm injection with profound teratospermia. Fertil. Steril., 2004, 82 : 847-849.

32. MENKVELD R., KRUGER T.F. : Advantages of strict (Tygerberg) criteria for evaluation of sperm morphology. Int. J. Androl., 1995, 18 Suppl $2: 36-42$.

33. MORTIMER D., MENKVELD R. : Sperm morphology assessment historical perspectives and current opinions. J. Androl., 2001, 22 : 192205.

34. PEER S., ELTES F., BERKOVITZ A., YEHUDA R., ITSYKSON P., BARTOOV $B$. : Is fine morphology of the human sperm nuclei affected by in vitro incubation at 37 degrees C ? Fertil. Steril., 2007 May 2 [Epub ahead of print].

35. RAJAK., FRANKEN D.R. : The role of paternal chromosomes and sperm morphology on the outcome of intracytoplasmic sperm injection. Andrologia, 2006, $38: 179-185$

36. SALUMETSA., SUIKKARI A.M., MOLS T., SODERSTROM-ANTTILA V., TUURI T. : Influence of oocytes and spermatozoa on early embryonic development. Fertil. Steril., 2002, 78 : 1082-1087.

37. SERMONDADE N., VIALARD F., BERGERE M. et al. : Evaluation de l'apport de la méthode d'observation des spermatozoïdes à fort grossissement en ICSI. Andrologie, 2007, $17: 212-221$.

38. SLAMAR., EUSTACHE F., DUCOT B. et al. : Time to pregnancy and semen parameters : a cross-sectional study among fertile couples from four European cities. Hum. Reprod., 2002, 17 : 503-515.

39. VAN WAART J., KRUGER T.F., LOMBARD C.J., OMBELET W. : Predictive value of normal sperm morphology in intrauterine insemination (IUI) : a structured literature review. Hum. Reprod. Update, 2001, $7: 495-500$

40. VAZQUEZ L., GOLDBERG F., DES JARLAIS N. : Papanicolaou and Kruger assessement of sperm morphology. Int. J. Androl., 1998, 21: 327-331.

41. VICARI E., DE PALMAA., BURRELLO N. et al. : Absolute polymorphic teratozoospermia in patients with oligo-asthenozoospermia is associated with an elevated sperm aneuploidy rate. J. Androl., 2003 , 24 : 598-603. 
42. WORLD HEATH ORGANISATION. WHO Laboratory Manual for the examination oh human Semen and Semen cervical Mucus Interaction, $2^{\text {nd }}$ edition Cambridge, United Kingdom : Cambridge University Press, 1987

43. WORLD HEATH ORGANISATION. WHO Laboratory Manual for the examination oh human Semen and Semen cervical Mucus Interaction, $3^{\text {rd }}$ edition Cambridge, United Kingdom : Cambridge University Press, 1992.

44. WORLD HEATH ORGANISATION. WHO Laboratory Manual for the examination oh human Semen and Semen cervical Mucus interaction, $4^{\text {th }}$ edition Cambridge, United Kingdom : Cambridge University Press, 1999.

Manuscrit reçu : novembre 2007 ; accepté février 2008.

\section{ABSTRACT}

Sperm morphology examination

Rim SAIIDI, Elodie GRUEL, Julie ROSET-BLESSMAN, Nathalie MOUSSET-SIMÉON, Sandrine MENON, Bertrand MACÉ, Nathalie RIVES

Semen analysis, as defined by World Health Organisation (WHO), is a fundamental step in the work-up of infertile couples. Spermatozoa morphology has been recognised as the best predictive factor in natural fertility, and in intrauterine insemination and classical in vitro fertilisation.

Ultrastructural spermatozoa abnormalities are the only sperm alterations likely to influence the outcome of ICSI. However, these abnormalities cannot be detected by conventional microscopy ( $¥ 100$ or $¥ 200-400$ ). Bartoov et al. (2002) developed a real-time spermatozoa observation system using a $¥ 6600$ magnification called MSOME (Magnification Motile Sperm Morphology Examination). Spermatozoa abnormalities detected with this technique are vacuoles localised on spermatozoa heads with variable number, size and site (nucleus or acrosome).

Spermatozoa evaluation using MSOME could be performed to predict the probability of fertilisation by these spermatozoa either spontaneously or after assisted reproductive technology.

Key words : infertility, morphology, spermatozoa, tératozoospermie 